\title{
Holonomy in the quantum navigation problem
}

\author{
B. Hubicska ${ }^{1}$ Z. Muzsnay ${ }^{1}$
}

Received: 18 February 2019 / Accepted: 28 August 2019 / Published online: 5 September 2019 (c) The Author(s) 2019

\begin{abstract}
In this paper, we investigate the holonomy structure of the Quantum Zermelo navigational problem introduced by Russel and Stepney (Phys Rev A 90:012303, 2014). We show that the curvature algebra describing infinitesimally the non-commutativity of parallel translation is infinite dimensional. Consequently, we obtain that the holonomy group cannot be a finite-dimensional group. These results demonstrate the deep difference between the Riemannian and Finslerian model and show that new phenomena occur.
\end{abstract}

Keywords Quantum Zermelo navigation · Riemann and Finsler geometry · Holonomy · Curvature

\section{Introduction}

In recent years, more and more focus tends to the applications of Finsler geometry in several fields of natural sciences. From general relativity through wildfire spread and seismic ray modeling to quantum mechanics, a lot of different areas take advantage of the tools of Finsler geometry $[15,18]$. The investigations of B. Russel and S. Stepney in $[19,20]$ opened a new way for the geometric investigations of several interesting phenomena in Quantum Information Processing (QIP) by the application of Bao et al. [2] theorem on the one-to-one correspondence between the solutions of the Zermelo navigational problem and Randers metrics. The holonomy group is a very natural geometric object. In a broader sense, it is a geometric concept which refers to the property that if we transport an object along a closed path, it may not return to its

This work is partially supported by the EFOP-3.6.2-16-2017-00015 Project, by the EFOP-3.6.1-16-2016-00022 Project and the 307818 TKA-DAAD exchange Project.

\footnotetext{
$\bowtie \quad$ B. Hubicska

hubicska.balazs@science.unideb.hu

Z. Muzsnay

muzsnay@science.unideb.hu

1 Institute of Mathematics, University of Debrecen, Pf 400, Debrecen 4002, Hungary
} 
original state. This phenomenon occurs in different areas of physics such as string theory [11] and what before the middle of the twentieth century was not considered as a geometric theory: quantum mechanics. The first appearance of the so-called quantum adiabatic holonomy was during the investigations of the dynamical Jahn-Teller effect [12]. Berry [4] showed that the adiabatic phase is a general phenomenon, and as pointed out by Simon [21], the celebrated Berry phase is an example of holonomy. The appearance of Randers manifolds, which are special types of Finsler manifolds, in QIP together with the method for realizing quantum computation through holonomy transformations proposed by Zanardi and Rasetti [23] served as a base motivation for us to start investigating Finslerian holonomy groups in a quantum mechanical setting.

In geometry, the holonomy group is the transformation group generated by parallel translations along closed curves with respect to a canonical connection. In the $n$ dimensional Riemannian case, the connection is the Levi-Civita connection and the generated holonomy group is a subgroup of the orthogonal group $O(n)$ [5]. In the Finslerian setting, the canonical connection is a homogeneous (but not necessarily linear) and norm-preserving (but not necessarily metric) connection $[1,10]$. In contrast to the Riemannian case, in which the complete classification of holonomy groups is known [3], there are only partial results about the holonomy groups of Finsler manifolds $[13,17,22]$. In $[14,16]$, a method has been developed to investigate the holonomy, which consists of considering the tangent structure of the holonomy groups, called the holonomy algebra. The holonomy algebra can offer important information on the holonomy group and give also the possibility to determine the holonomy group even in some infinite-dimensional cases [13,17].

In this paper, we show a new non-trivial application of this theory in Quantum Mechanics by using the 2-dimensional Quantum Zermelo problem introduced in [7]. By investigating the curvature algebra describing infinitesimally the noncommutativity of parallel translations, we prove that its dimension is infinite. Consequently, we obtain that the holonomy group cannot be a finite-dimensional group. This result demonstrates the radical difference between the Riemannian and Finslerian model and shows that with respect to the classical Riemannian settings, a new phenomenon occurs: the presence of an ineliminable force extends hugely the set of holonomy transformations and therefore the set of states realizable from one given state.

\section{Preliminaries}

\subsection{Randers model of quantum information processing}

In a closed finite-dimensional quantum system, the state space is $\mathbb{C}^{n}$ for some $n \in \mathbb{N}$ and the physical states can be identified with the rays of this space, that is the projective space $\mathbb{C} P^{n-1}$ of the underlying Hilbert space. In QIP, the task of the "navigator" is to find the shortest path from an initial state $\left|\Psi_{I}\right\rangle$ to a final state $\left|\Psi_{F}\right\rangle$. This means that one have to find the geodesics of the corresponding Riemannian manifold. The situation is slightly changed if we take into account an ineliminable force on the manifold, which can be represented as a vector field also referred to as the "wind". This formulation 
indicates that this problem is equivalent to the Zermelo navigational problem on a Riemannian manifold. In [2], the authors proved that the problem of finding the shortest path (in terms of time) in this setting is equivalent to that of finding the geodesics of a suitable Randers manifold, which is a special kind of Finsler manifold. The problem is hard to solve on the level of state space, but in [7] and in [6] the authors showed that one can lift this problem to the space acting on the states: the special unitary group $\operatorname{SU}(n)$. The task is then to find a control Hamiltonian $\hat{H}_{c}(t)$ in the Lie algebra $\mathfrak{s u}(n)$ of SU(n) which together with a time-independent Hamiltonian $\hat{H}_{0}$, representing the effect of the ineliminable external field, forms $\hat{H}(t)=\hat{H}_{c}(t)+\hat{H}_{0}$ and generates the evolution of our initial state to the final state. The physical motivation and explanation can be found in [7]. Here, we are focusing on the geometric background of the Finslerian model. Geometric aspects and elementary solution to the time-independent quantum navigation problem can be found in [8].

Let $M$ be an $n$-dimensional manifold, $T M$ its tangent manifold, $\pi: T M \rightarrow M$ the canonical projection. Local coordinates $\left(x^{i}\right)$ on $M$ induce local coordinates $\left(x^{i}, y^{i}\right)$ on $T M$ where $y=y^{i} \frac{\partial}{\partial x^{i}}$.

\subsection{Finsler metric}

A function $\mathcal{F}: T M \rightarrow \mathbb{R}_{+}$is called the Finsler (norm) function, if it is a positively 1-homogeneous continuous function, smooth on $T M \backslash\{0\}$ and

$$
g_{i j}=\frac{1}{2} \frac{\partial^{2} E}{\partial y^{i} \partial y^{j}},
$$

is positive definite at every $y \in T_{x} M \backslash\{0\}$, where $E:=\frac{1}{2} \mathcal{F}^{2}$ is the energy function of $\mathcal{F}$. Formally, the Finsler metric $g=g_{i j} \mathrm{~d} x^{i} \otimes \mathrm{d} x^{j}$ on $M$ is the same as a Riemannian metric, except that the coefficients $g_{i j}$ can depend on the $y$ variable, that is on the direction too. The hypersurface of $T_{x} M$ defined by

$$
\mathcal{I}_{x}=\left\{y \in T_{x} M: \mathcal{F}(y)=1\right\} .
$$

is called the indicatrix at $x \in M$. The geodesics of $(M, \mathcal{F})$ are given by the solutions of a system of second order ordinary differential equations

$$
\ddot{x}^{i}=f^{i}(x, \dot{x})
$$

in a local coordinate system, where $f^{i}(x, y)$ are determined by the formula

$$
f^{i}(x, y)=g^{i l}\left(\frac{1}{2} \frac{\partial g_{j k}}{\partial x^{l}}-\frac{\partial g_{j l}}{\partial x^{k}}\right) y^{j} y^{k}, \quad i=1, \ldots, n .
$$

One of the most accessible and demonstrative Finsler metrics comes from Zermelo's navigation problem where the geodesics corresponds to the paths of shortest travel time in a Riemannian manifold $(M, h)$, under the influence of a wind or a current which is 
represented by a vector field $W$. The geometry can be described in terms of a Finslerian setting with the Finsler function

$$
\mathcal{F}(x, y)=\frac{\sqrt{h_{x}(W, y)^{2}+h_{x}(y, y)\left(1-h_{x}(W, W)\right)}-h_{x}(W, y)}{1-h_{x}(W, W)}
$$

where $h_{x}(W, W)<1$. As the results of [2] show, there is a one-to-one correspondence between the solutions of the Zermelo navigational problem and Randers metrics.

\subsection{Covariant derivative and parallel translation}

A vector field $X=X^{i}(t) \frac{\partial}{\partial x^{i}}$ along a curve $c:[0,1] \rightarrow M$ is called parallel if $D_{\dot{c}} X(t)=0$ where the covariant derivative is defined as

$$
D_{\dot{c}} X=\left(\frac{\mathrm{d} X^{i}}{\mathrm{~d} t}+G_{j}^{i}(c, X) \dot{c}^{j}\right) \frac{\partial}{\partial x^{i}},
$$

with $G_{j}^{i}=-\frac{1}{2} \frac{\partial f^{i}}{\partial y^{j}}$. For any $X_{0} \in T_{c_{o}} M$, there exists a unique parallel vector field $X(t)$ along the curve $c$ such that $X_{0}=X(0)$. One can easily see, that if $X(t)$ is a parallel vector field along $c$, then $\lambda X(t)$ is also parallel along $c$ for any $\lambda \geq 0$. In this way, the homogeneous parallel translation along a curve $c$ is defined by the positive homogeneous map $\mathcal{P}_{c}^{t}$ given by $\mathcal{P}_{c}^{t}\left(X_{0}\right)=X(t)$. Since the parallel translation preserves the Finslerian norm, it can be considered as a map between the indicatrices

$$
\mathcal{P}_{c}^{t}: \mathcal{I}_{c_{o}} \rightarrow \mathcal{I}_{c_{t}}
$$

The parallel translation on a Finsler manifold can also be introduced by considering the associated Ehresmann connection: the horizontal distribution is determined by the image of the horizontal lift $T_{x} M \rightarrow T_{(x, y)} T M$ defined in the local basis as

$$
\left(\frac{\partial}{\partial x^{i}}\right)^{h}=\frac{\partial}{\partial x^{i}}-G_{i}^{k}(x, y) \frac{\partial}{\partial y^{k}},
$$

where $y \in T_{x} M$. We have the decomposition $T_{y} T M=\mathcal{H}_{y} \oplus \mathcal{V}_{y}$, where $\mathcal{V}=\operatorname{ker} \pi_{*}$ is the vertical distribution. The corresponding projectors are denoted by $h$ and $v$. The horizontal lift of a curve $c:[0,1] \rightarrow M$ with initial condition $X_{0} \in T_{c_{0}} M$ is a curve $c^{h}:[0,1] \rightarrow T M$ such that $\pi \circ c^{h}=c, \frac{\mathrm{d} c^{h}}{\mathrm{~d} t}=\left(\frac{\mathrm{d} c}{\mathrm{~d} t}\right)^{h}$ and $c^{h}(0)=X_{0}$.

Then the parallel translation can be geometrically obtained as $\mathcal{P}_{c}^{t}\left(X_{0}\right)=c^{h}(t)$. The horizontal distribution $\mathcal{H} T M$ is, in general, non-integrable. The obstruction to its integrability is given by the curvature tensor $R=\frac{1}{2}[h, h]$ which is the Nijenhuis torsion of the horizontal projector [10]. The curvature tensor field is defined by $R=$ $R_{j k}^{i}(x, y) \mathrm{d} x^{j} \otimes \mathrm{d} x^{k} \otimes \frac{\partial}{\partial y^{i}}$ where

$$
R_{j k}^{i}=\frac{\partial G_{j}^{i}}{\partial x^{k}}-\frac{\partial G_{k}^{i}}{\partial x^{j}}+G_{j}^{m} G_{k m}^{i}-G_{k}^{m} G_{j m}^{i}
$$

in a local coordinate system. 


\subsection{Holonomy group, holonomy algebra, curvature algebra}

The holonomy group $\operatorname{Hol}_{x}(M)$ of a Finsler manifold $(M, \mathcal{F})$ at a point $x \in M$ is the group generated by parallel translations along piece-wise differentiable loops starting and ending at $x$. Considering the parallel translation (4) on the indicatrix $\mathcal{I}_{x}$, a holonomy element is a diffeomorphism $\mathcal{P}_{c}: \mathcal{I}_{x} \rightarrow \mathcal{I}_{x}$, and therefore the holonomy group can be considered as a subgroup of the diffeomorphism group of the indicatrix $\mathcal{I}_{x}$ :

$$
\mathcal{H o l}_{x}(M) \subset \operatorname{Diff}^{\infty}\left(\mathcal{I}_{x}\right)
$$

The tangent Lie algebra $\mathfrak{h o l}_{x}(M)$ of the holonomy group is called the holonomy algebra [14]. Any Lie subalgebra of $\mathfrak{h o l}_{x}(M)$ inherits its tangential property. In particular, the curvature algebra $\mathfrak{R}_{x}$, generated by vector fields in the image of the curvature tensor, is a Lie subalgebra of $\mathfrak{h o l}_{x}(M)$. Consequently, the element of the curvature algebra is tangent to the holonomy group $\mathcal{H o l}_{x}(M)$. At the level of Lie algebras, we have

$$
\mathfrak{R}_{x} \subset \mathfrak{h o l}_{x}(M) \subset \mathfrak{X}^{\infty}\left(\mathcal{I}_{x}\right)
$$

and therefore at the level of groups, we get

$$
\exp \left(\Re_{x}\right) \subset \exp \left(\mathfrak{h o l}_{x}(M)\right) \subset \mathcal{H}^{c} l_{x}^{c}(M) \subset \mathcal{D i f f}{ }^{\infty}\left(\mathcal{I}_{x}\right)
$$

where $\mathcal{H}^{c} l_{x}^{c}(M)$ denotes the topological closure of the holonomy group with respect to the $C^{\infty}$ topology of $\mathcal{D}$ iff ${ }^{\infty}\left(\mathcal{I}_{x}\right)$. More about the tangent Lie algebras of diffeomorphism groups and about the holonomy group and holonomy algebra can be found in [14].

\section{Holonomy of quantum information processing in Randers model}

\subsection{2-Dimensional Quantum Zermelo problem}

We consider a specific case of the two-state quantum system: a single spin particle in a magnetic field. The manifold is SU(2) and its tangent space can be identified with $\mathfrak{s u}(2)$, which is the 3-dimensional Lie algebra of $2 \times 2$ Hermitian matrices. As in [7], we consider an invariant "wind" vector field, represented in the Lie algebra by $\hat{H}_{0}$ and the invariant Riemannian metric coming from the Killing form:

$$
h(\hat{A}, \hat{B}):=\operatorname{tr}\left(\hat{A}^{\dagger} \hat{B}\right)
$$

$\hat{A}, \hat{B} \in \mathfrak{s u}(2)$. The Lie algebra $\mathfrak{s u}(2)$ is spanned by $\hat{E}_{1}=i \sigma_{1}, \hat{E}_{2}=-i \sigma_{2}, \hat{E}_{3}=i \sigma_{3}$ where the sigmas are the Pauli matrices:

$$
\sigma_{1}=\left(\begin{array}{ll}
0 & 1 \\
1 & 0
\end{array}\right), \quad \sigma_{2}=\left(\begin{array}{cc}
0 & -i \\
i & 0
\end{array}\right), \quad \sigma_{3}=\left(\begin{array}{cc}
1 & 0 \\
0 & -1
\end{array}\right)
$$


We will work with the coordinates $(x, \xi)$ on the tangent bundle, where $(x)=$ $\left(x_{1}, x_{2}, x_{3}\right)$ are coordinates on the group $\mathrm{SU}(2)$ and $(\xi)=\left(\xi_{1}, \xi_{2}, \xi_{3}\right)$ are the invariant coordinates in the Lie algebra $\mathfrak{s u}(2)$ with respect to the basis $\mathcal{E}:=\left\{\hat{E}_{1}, \hat{E}_{2}, \hat{E}_{3}\right\}$. The relation between the standard coordinates and the invariant coordinates on $T G=$ $G \times \mathfrak{g}$ can be found by $\rho_{x, *}^{-1}(x, y)=(x, \xi)$, where $\rho: \mathrm{SU}(2) \rightarrow \mathrm{SU}(2)$ is the right translation. Modulo a rigid transformation, we can suppose that $\hat{H}_{0}=c \hat{E}_{1}$ with $c \in \mathbb{R}$. With (8) and $W=\hat{H}_{0}$, one can calculate (2) and find the corresponding invariant Randers metric:

$$
\mathcal{F}(\xi)=\frac{1}{1-2 c^{2}}\left(\alpha_{\xi}+\beta_{\xi}\right)
$$

with a Riemannian norm $\alpha$ and a $1-$ form $\beta$ :

$$
\alpha_{\xi}:=\sqrt{2 \xi_{1}^{2}+2 \xi_{2}^{2}+2 \xi_{3}^{2}-4 \xi_{2}^{2} c^{2}-4 \xi_{3}^{2} c^{2}}, \quad \beta_{\xi}:=-2 c \xi_{1} .
$$

For simplicity, the 3-dimensional Finsler space corresponding to the 2-dimensional Quantum Zermelo problem will be denoted by $\mathcal{Q}=(\mathrm{SU}(2), \mathcal{F})$.

We note that the indicatrix (1) is

$$
\mathcal{I}=\left\{\xi \in \mathfrak{s u}(2): \alpha_{\xi}=1+2 c \xi_{1}-2 c^{2}\right\}
$$

a two-dimensional compact manifold. Let $E:=\frac{1}{2} \mathcal{F}^{2}$ be the energy function. The invariant formulation of the Euler-Lagrange equations, called the Euler-Poincaré equations

$$
\frac{d}{d t} \frac{\partial E}{\partial \xi}=-a d_{\xi}^{*}\left(\frac{\partial E}{\partial \xi}\right),
$$

can be used to determine the geodesic equation on the Lie algebra [9]. Using the basis $\mathcal{E}=\left\{\hat{E}_{1}, \hat{E}_{2}, \hat{E}_{3}\right\}$. the equations (12) take the form

$$
\frac{\mathrm{d}}{\mathrm{d} t} \frac{\partial E}{\partial \xi_{d}}=-C_{a d}^{b} \frac{\partial E}{\partial \xi_{b}} \xi_{a}
$$

where $C_{a d}^{b}$ are the structure constants with respect to the basis $\mathcal{E}$ : The nonzero terms are determined by $C_{12}^{3}=C_{23}^{1}=C_{31}^{2}=2$. We get that

$$
\dot{\xi}_{1}=0, \quad \dot{\xi}_{2}=-2 c \xi_{3} \mathcal{F}(\xi), \quad \dot{\xi}_{3}=2 c \xi_{2} \mathcal{F}(\xi)
$$




\subsection{Curvature algebra and holonomy}

The curvature tensor can be obtained from the spray coefficients. The restriction of the curvature vector fields $R\left(\hat{E}_{i}, \hat{E}_{j}\right)$ on the indicatrix $(11)$ is

$$
\begin{array}{lll}
R_{1}:=\left.R\left(\hat{E}_{1}, \hat{E}_{2}\right)\right|_{\mathcal{I}}=-\frac{\xi_{2}}{\alpha_{\xi}} \partial_{\xi_{1}}+\frac{\left(\xi_{1}-c\right)}{\alpha_{\xi}} \partial_{\xi_{2}} & \in \mathfrak{X}^{\infty}(\mathcal{I}), \\
R_{2}:=\left.R\left(\hat{E}_{1}, \hat{E}_{3}\right)\right|_{\mathcal{I}}=-\frac{\xi_{3}}{\alpha_{\xi}} \partial_{\xi_{1}}+\frac{\left(\xi_{1}-c\right)}{\alpha_{\xi}} \partial_{\xi_{3}} & \in \mathfrak{X}^{\infty}(\mathcal{I}), \\
R_{3}:=\left.R\left(\hat{E}_{2}, \hat{E}_{3}\right)\right|_{\mathcal{I}}=-\frac{\xi_{3}}{\alpha_{\xi}} \partial_{\xi_{2}}+\frac{\xi_{2}}{\alpha_{\xi}} \partial_{\xi_{3}} & \in \mathfrak{X}^{\infty}(\mathcal{I}),
\end{array}
$$

where $\alpha_{\xi}$ is defined in (10). The curvature algebra $\Re$ is generated by the commutators of the vector fields $R_{i}, i=1,2,3$, that is:

$$
\Re=\left\langle R_{1}, R_{2}, R_{3}\right\rangle_{\text {Lie }} .
$$

It is not difficult to calculate the first Lie brackets of the curvature vector fields:

$$
\left[R_{1}, R_{3}\right]=\frac{1}{\alpha_{\xi}} R_{2}+\frac{2 c \xi_{2}}{\alpha_{\xi}^{2}} R_{3}, \quad\left[R_{2}, R_{3}\right]=\frac{-1}{\alpha_{\xi}} R_{1}+\frac{2 c \xi_{3}}{\alpha_{\xi}^{2}} R_{3} .
$$

More generally, we have the following:

Lemma 3.1 Let $L_{0}=R_{2}$ and denote $L_{k}=\left[L_{k-1}, R_{3}\right]$ the Lie bracket of the vector field $L_{k-1}$ and $R_{2}$ for $k \geq 1$. Then

$$
L_{k}=\left\{\begin{array}{cc}
\frac{\epsilon_{k}}{\alpha_{\xi}^{k}} R_{2}+2 k c \frac{\epsilon_{k} \xi_{2}}{\alpha_{\xi}^{k+1}} R_{3}, & \text { if } k \equiv 0 \bmod 2, \\
\frac{\epsilon_{k}}{\alpha_{\xi}^{k}} R_{1}-2 k c \frac{\epsilon_{k} \xi_{3}}{\alpha_{\xi}^{k+1}} R_{3}, & \text { if } k \equiv 1 \bmod 2,
\end{array}\right.
$$

where $\epsilon_{k}=-1$, if $k=4 l+1$ or $k=4 l+2$ and $\epsilon_{k}=1$, if $k=4 l+3$ or $k=4 l$ for some $l \in \mathbb{N}$.

Proof We prove the lemma by recurrence. Using the fact that the curvature vector fields (15) are tangent to the indicatrix (11), one can find for odd $k$ :

$$
\begin{aligned}
{\left[L_{k}, R_{3}\right] } & =\left[\frac{\epsilon_{k}}{\alpha_{\xi}^{k}} R_{1}, R_{3}\right]-\left[\frac{\epsilon_{k} 2 k c \xi_{3}}{\alpha_{\xi}^{k+1}} R_{3}, R_{3}\right] \\
& =\frac{\epsilon_{k}}{\alpha_{\xi}^{k+1}}\left(R_{2}+\frac{2 c \xi_{2}}{\alpha_{\xi}} R_{3}\right)+\frac{\epsilon_{k} 2 k c \xi_{2}}{\alpha_{\xi}^{k+2}} R_{3} \\
& =\frac{\epsilon_{k+1}}{\alpha_{\xi}^{k+1}} R_{2}+2(k+1) c \frac{\epsilon_{k+1} \xi_{2}}{\alpha_{\xi}^{k+2}} R_{3}=L_{k+1},
\end{aligned}
$$


where we used that $\epsilon_{k+1}=\epsilon_{k}$ when $k$ is odd. For even $k$, we have analogous computation:

$$
\begin{aligned}
{\left[L_{k}, R_{3}\right] } & =\left[\frac{\epsilon_{k}}{\alpha_{\xi}^{k}} R_{2}, R_{3}\right]+\left[\frac{\epsilon_{k} 2 k c \xi_{2}}{\alpha_{\xi}^{k+1}} R_{3}, R_{3}\right] \\
& =\frac{\epsilon_{k}}{\alpha_{\xi}^{k+1}}\left(-R_{1}+\frac{2 c \xi_{3}}{\alpha_{\xi}} R_{3}\right)+\frac{\epsilon_{k} 2 k c \xi_{3}}{\alpha_{\xi}^{k+2}} R_{3} \\
& =\frac{\epsilon_{k+1}}{\alpha_{\xi}^{k+1}} R_{1}-2(k+1) c \frac{\epsilon_{k+1} \xi_{3}}{\alpha_{\xi}^{k+2}} R_{3}=L_{k+1}
\end{aligned}
$$

where we used that $\epsilon_{i+1}=-\epsilon_{i}$ when $k$ is even.

Proposition 3.2 In the presence of external wind $W$, the curvature algebra $\mathfrak{R}$ of the Finsler metric (9) is an infinite-dimensional Lie subalgebra of $\mathfrak{X}^{\infty}(\mathcal{I})$ of smooth vector fields of the indicatrix (11).

Proof By definition, the curvature algebra $\mathfrak{R}$ is the Lie subalgebra of $\mathfrak{X}^{\infty}(\mathcal{I})$ generated by the curvature vector fields [14, Definition 4.10]. In the case of the Finsler metric (9), the curvature algebra (16) is generated by the three curvature vector fields (15) and by their successive Lie brackets. If the external wind $W$ is nonzero, then $c \neq 0$ and, using the notation of Lemma 3.1, we have $L_{k} \in \mathfrak{R}$ for any $k \in \mathbb{N}$. As the formulas of (18) show, every $L_{k}$ can be expressed by some combination of the curvature vector fields, but the coefficients are polynomials with increasing degree in each step. Consequently,

$$
\left\{L_{k} \mid k \in \mathbb{N}\right\} \subset \Re\left(\subset \mathfrak{X}^{\infty}(\mathcal{I})\right)
$$

is an $\mathbb{R}$-linearly independent infinite set of vector fields in the curvature algebra, that is $\mathfrak{R}$ is an infinite-dimensional Lie subalgebra of $\mathfrak{X}^{\infty}(\mathcal{I})$.

We remark that if there is no external wind, that is $c=0$, then (9) is a Riemann metric: On the indicatrix, we have $\alpha_{\xi}=1$ and one can easily see that $\Re$ is isomorphic to $\mathfrak{s o}(3)$.

Theorem 3.3 The holonomy group $\mathcal{H o l}(\mathcal{Q})$ of the 2-dimensional Quantum Zermelo problem in the presence of an external wind $W$ is not a finite-dimensional Lie group.

Proof We do not know at the moment if the group $\mathcal{H o l}(\mathcal{Q})$ has a smooth Lie group structure or not, but we do know that it is a subgroup of $\mathcal{D}$ if $f^{\infty}(\mathcal{I})$, the diffeomorphism group of the indicatrix $\mathcal{I}$. With $\mathcal{I}$ being compact, the tangent space of the group $\mathcal{H} \operatorname{ol}(\mathcal{Q})$ is a Lie subalgebra of $\mathfrak{X}^{\infty}(\mathcal{I})$, see [14, Sec. 4]. The tangent algebra of the holonomy group $\operatorname{Hol}(\mathcal{Q})$ is the holonomy algebra $\mathfrak{h o l}(\mathcal{Q})$, and as (6) shows, it contains the curvature algebra $\mathfrak{R}$. As Proposition 3.2 shows, $\Re$ is infinite dimensional, and therefore $\mathfrak{h o l}(\mathcal{Q})$ is also an infinite-dimensional Lie algebra. Consequently, from (7) we get that the holonomy group $\mathcal{H o l}(\mathcal{Q})$ cannot be a finite-dimensional Lie group.

We remark that if there is no external wind $W$, then $\mathcal{Q}=(\operatorname{SU}(2), \alpha)$ is a 3-dimensional Riemannian manifold and its holonomy group $\mathcal{H o l}(\mathcal{Q})$ is the 3dimensional special orthogonal group $S O(3)$. 


\section{Conclusion}

Theorem 3.3 demonstrates clearly the deep difference between the Riemannian and Finslerian model: in the Riemannian setting, the holonomy group is necessarily a finite-dimensional Lie group. But using the Finslerian model in the presence of a magnetic field, this is no longer true: the holonomy group can be a much larger group. Indeed, there are examples, where the topological closure of the holonomy group is the diffeomorphism group of the indicatrix $[13,17]$. Until now, results on the holonomy are obtained on cases, where the parallel translations are linear (thoroughly analyzed by Szabó [22]) or the flag-curvature is constant. We note that in the 2-dimensional Quantum Zermelo problem considered above the flag-curvature is not constant: with an explicit computation one can show that $\mathcal{Q}$ has a non-constant scalar flag-curvature.

Acknowledgements Open access funding provided by University of Debrecen (DE).

Open Access This article is distributed under the terms of the Creative Commons Attribution 4.0 International License (http://creativecommons.org/licenses/by/4.0/), which permits unrestricted use, distribution, and reproduction in any medium, provided you give appropriate credit to the original author(s) and the source, provide a link to the Creative Commons license, and indicate if changes were made.

\section{References}

1. Bao, D., Chern, S., Shen, Z.: An Introduction to Riemann-Finsler Geometry. Graduate Texts in Mathematics. Springer, New York (2000)

2. Bao, D., Robles, C., Shen, Z.: Zermelo navigation on Riemannian manifolds. J. Differ. Geom. 66(3), 377-435 (2004)

3. Berger, M.: Sur les groupes d'holonomie homogène des variétés à connexion affine et des variétés riemanniennes. Bull. Soc. Math. France 83, 279-330 (1955)

4. Berry, M.V.: Quantal phase factors accompanying adiabatic changes. Proc. R. Soc. Lond. A392, 45-57 (1984)

5. Borel, A., Lichnerowicz, A.: Groupes d'holonomie des variétés riemanniennes. C. R. Acad. Sci. Paris 234, 1835-1837 (1952)

6. Brody, C., Gibbons, W., Meier, D.: Time-optimal navigation through quantum wind. New J. Phys. 17, 033048 (2015)

7. Brody, D.C., Meier, D.: Solution to the Quantum Zermelo navigation problem. Phys. Rev. Lett. 114, 100502 (2015)

8. Brody, D.C., Meier, D.: Elementary solution to the time-independent quantum navigation problem. J. Phys. A Math. Theor. 48, 055302 (9pp) (2015)

9. Holm, D.D., Marsden, E., Ratiu, S.: The euler-poincaré equations and semidirectproducts with applications to continuum theories. Adv. Math. 137(1), 1-81 (1998)

10. Grifone, J.: Structure presque-tangente et connexions. I. Ann. Inst. Fourier (Grenoble) 22(1), 287-334 (1972)

11. Gubser, S.: Tasi lectures: special holonomy in string theory and m-theory. CALT-68-2370 (2002)

12. Longuet-Higgins, C.H.C., Öpik, U., Pryce, L., Sack, A.: Studies of the Jahn-Teller Effect. II. The dynamical problem. Proc. R. Soc. Lond. A244, 1-16 (1958)

13. Hubicska, B., Muzsnay, Z.: The holonomy groups of projectively flat Randers two-manifolds of constant curvature (2018), arXiv:1805.05216

14. Hubicska, B., Muzsnay, Z.: Tangent lie algebra of a diffeomorphism group and application to holonomy theory. J. Geom. Anal. (2019, Jan accepted). arXiv:1805.05265

15. Markvorsen, S.: A finsler geodesic spray paradigm for wildfire spread modelling. Nonlinear Anal. 28, 208-228 (2016) 
16. Muzsnay, Z., Nagy, P.T.: Tangent Lie algebras to the holonomy group of a Finsler manifold. Commun. Math. 19(2), 137-147 (2011)

17. Muzsnay, Z., Nagy, P.T.: Finsler 2-manifolds with maximal holonomy group of infinite dimension. Differ. Geom. Appl. 39, 1-9 (2015)

18. Peter, M.A.S., Antonelli, L., Bóna, A.: Seismic rays as finsler geodesics. Nonlinear Anal. 4, 711-722 (2003)

19. Russel, B., Stepney, S.: Zermelo navigation and a speed limit to quantum information processing. Phys. Rev. A 90, 012303 (2014)

20. Russel, B., Stepney, S.: Zermelo navigation in the quantum brachistochrone. J. Phys. A 48, 313 (2015)

21. Simon, B.: Holonomy, the quantum Adiabatic theorem, and Berry's phase. Phys. Rev. Lett. 51, 2167 (1983)

22. Szabó, Z.I.: Positive definite Berwald spaces. Structure theorems on Berwald spaces. Tensor (N.S.) 35(1), 25-39 (1981)

23. Zanardi, P., Rasetti, M.: Holonomic quantum computation. Phys. Lett. A. 264, 94-99 (1999)

Publisher's Note Springer Nature remains neutral with regard to jurisdictional claims in published maps and institutional affiliations. 